\title{
Photometry and spectroscopy of the new symbiotic star 2SXPS J173508.4-292958
}

\author{
U. Munari ${ }^{1}$, P. Valisa ${ }^{2}$, A. Vagnozzi ${ }^{2}$, S. Dallaporta ${ }^{2}$, \\ F.-J. Hambsch ${ }^{2}$ and A. Frigo ${ }^{2}$ \\ 1 INAF Padova Astronomical Observatory, I-36012 Asiago (VI), Italy \\ 2 ANS Collaboration, Astronomical Observatory, 36012 Asiago (VI), Italy
}

Received: December 2, 2020; Accepted: December 21, 2020

\begin{abstract}
We present and discuss the results of our photometric and spectroscopic monitoring of 2SXPS J173508.4-292958 carried out from April to August 2020. This X-ray source, in the foreground with respect to the Galactic center, brightened in X-rays during 2020, prompting our follow-up optical observations. We found the star to contain a K4III giant with a modest but highly variable $\mathrm{H} \alpha$ emission, composed by a $\sim 470 \mathrm{~km} \mathrm{~s}^{-1}$ wide component with superimposed a narrow absorption, offset by a positive velocity with respect to the giant. No orbital motion is detected for the K4III, showing an heliocentric radial velocity stable at $-12 \pm 1 \mathrm{~km} \mathrm{~s}^{-1}$. No flickering in excess of $0.005 \mathrm{mag}$ in $B$ band was observed at three separate visits of 2SXPS J173508.4292958. While photometrically stable in 2016 through 2018, in 2019 the star developed a limited photometric variability, that in 2020 took the form of a sinusoidal modulation with a period of 38 days and an amplitude of $0.12 \mathrm{mag}$ in $V$ band. We argue this variability cannot be ascribed to Roche-lobe filling by the K4III star. No correlation is observed between the photometric variability and the amount of emission in $\mathrm{H} \alpha$, the latter probably originating directly from the accretion disk around the accreting companion. While no emission from dust is detected at mid-IR wavelengths, an excess in $U$-band is probably present and caused by direct emission from the accretion disk. We conclude that 2SXPS J173508.4-292958 is a new symbiotic star of the accreting-only variety (AO-SySt).
\end{abstract}

Key words: binaries: symbiotic

\section{Introduction}

Attention to the X-ray source 2SXPS J173508.4-292958 has been recently driven by a report from Heinke et al. (2020) about a brightening of the object they observed in X-rays with the Swift satellite in April 2020. Noting the positional coincidence with the cool 2MASS star 17350831-2929580 of Ks=7.4 mag, Heinke et al. suggested that 2SXPS J173508.4-292958 could be a previously unknown symbiotic star. The optical counterpart was quickly subjected to BVRI photometry and high- and low-resolution spectroscopy by Munari et al. (2020a) that found 2SXPS J173508.4-292958 to host a K4 III/II giant, with marked 
near-UV excess and $\mathrm{H} \alpha$ in emission, the latter showing a broad profile with a central absorption. Such properties confirm a probable symbiotic nature for 2SXPS J173508.4-292958 (for a recent global review of symbiotic stars see Munari 2019). In this paper we report about the results of our follow-up photometric and spectroscopic campaign to monitor 2SXPS J173508.4-292958 (2SXPS for short in the rest of this paper) during the period of seasonal visibility in 2020.

\section{Observations}

Our observations have been carried out primarily from Italy. At such northern latitudes, the $-29^{\circ}$ declination of 2SXPS implies a very short observing window, $\sim 2$ hours around transit at meridian at just 15 to $20^{\circ}$ above horizon. Such a short observing interval coupled with the large atmospheric extinction and seeing degradation led to a lower-than-usual $\mathrm{S} / \mathrm{N}$ for our observations, and argued against employing the highest possible resolution in the spectroscopic observations.

\section{1. $B V R I$ photometry}

We have obtained optical photometry of 2SXPS in the Landolt photometric system from late April to early August 2020 with two telescopes operated by ANS Collaboration. ID 0310 is a $0.30 \mathrm{~m} \mathrm{f} / 8$ Richey-Chretien telescope located in Cembra (TN, Italy) and equipped with an SBIG ST- 8 CCD camera, $1530 \times 1020$ array, $9 \mu \mathrm{m}$ pixels $\equiv 0.77^{\prime \prime} /$ pix, with a field of view of $19^{\prime} \times 13^{\prime}$. ID 1301 is a $0.50 \mathrm{~m}$ $\mathrm{f} / 6$ Ritchey-Chretien telescope located in Stroncone (TR, Italy). It feeds light to an SBIG STL1001E CCD Camera $1024 \times 1024$ array, $24 \mu \mathrm{m}$ pixels $\equiv 1.60^{\prime \prime} /$ pix, field of view of $28^{\prime} \times 28^{\prime}$. Both telescopes adopt $B V R_{\mathrm{C}} I_{\mathrm{C}}$ photometric filters from Astrodon, in the version corrected for red-leak.

Data reduction has involved all the usual steps for bias, dark and flat with calibration images collected during the same observing nights. We adopted aperture photometry because the sparse field around 2SXPS did not required PSFfitting procedures. The transformation from the local to the Landolt standard system was carried out via color equations calibrated on a photometric sequence recorded on the same frames as 2SXPS:

$$
\begin{aligned}
V & =v+\alpha_{v} \times(v-i)+\gamma_{v} \\
B-V & =\beta_{b v} \times(b-v)+\delta_{b v} \\
V-R & =\beta_{v r} \times(v-r)+\delta_{v r} \\
V-I & =\beta_{v i} \times(v-i)+\delta_{v i}
\end{aligned}
$$

where lowercase and uppercase letters denote values in the local and and in the standard system, respectively. The local photometric sequence has been extracted from APASS DR8 survey (Henden et al. 2016), ported to the Landolt 
Table 1. Our BVRI photometry on the Landolt system of 2SXPS J173508.4-292958.

\begin{tabular}{|c|c|c|c|c|c|c|c|c|c|c|}
\hline HJD & date & B & err & V & err & $\mathrm{R}$ & err & I & err & ID \\
\hline 8962.57973 & $2020-04-23.080$ & 13.489 & 0.008 & 11.846 & 0.005 & 10.904 & 0.007 & 9.935 & 0.015 & 0310 \\
\hline 8962.57973 & $2020-04-23.080$ & 13.490 & 0.008 & 11.848 & 0.005 & 10.906 & 0.007 & 9.935 & 0.015 & 0310 \\
\hline 8963.57773 & $2020-04-24.078$ & 13.426 & 0.008 & 11.835 & 0.006 & 10.974 & 0.007 & 9.890 & 0.011 & 1301 \\
\hline 8963.58953 & $2020-04-24.090$ & 13.444 & 0.009 & 11.851 & 0.007 & 10.911 & 0.008 & 9.898 & 0.015 & 0310 \\
\hline 8971.58456 & $2020-05-02.085$ & 13.417 & 0.009 & 11.788 & 0.007 & 10.835 & 0.008 & 9.893 & 0.013 & 0310 \\
\hline 8974.57796 & $2020-05-05.078$ & 13.387 & 0.024 & 11.757 & 0.008 & 10.856 & 0.008 & 9.866 & 0.009 & 0310 \\
\hline 8978.53534 & 2020-05-09.035 & 13.376 & 0.010 & 11.780 & 0.005 & 11.174 & 0.005 & 0.103 & 0.005 & 1301 \\
\hline 8990.53842 & $2020-05-21.038$ & 13.466 & 0.008 & 11.840 & 0.007 & 10.924 & 0.008 & 9.936 & 0.012 & 0310 \\
\hline 8990.56051 & $2020-05-21.061$ & 13.442 & 0.017 & 11.861 & 0.008 & & & 9.913 & 0.012 & 0310 \\
\hline 8991.50080 & $2020-05-22.001$ & 13.452 & 0.006 & 11.843 & 0.004 & 10.917 & 0.003 & 9.903 & 0.008 & 1301 \\
\hline 8992.50328 & $2020-05-23.003$ & 13.512 & 0.007 & 11.859 & 0.003 & 10.917 & 0.004 & 9.893 & 0.009 & 1301 \\
\hline 8993.54900 & $2020-05-24.049$ & 13.496 & 0.007 & 11.871 & 0.004 & 10.927 & 0.004 & 9.912 & 0.011 & 1301 \\
\hline 8994.54993 & $2020-05-25.050$ & 13.444 & 0.013 & 11.852 & 0.006 & 10.955 & 0.010 & 9.944 & 0.008 & 0310 \\
\hline 8995.51675 & $2020-05-26.017$ & 13.486 & 0.009 & 11.901 & 0.008 & 10.908 & 0.008 & 9.944 & 0.012 & 0310 \\
\hline 8995.53700 & $2020-05-26.037$ & 13.469 & 0.013 & 11.837 & 0.008 & & & 9.945 & 0.010 & 0310 \\
\hline 8995.54481 & $2020-05-26.045$ & & & 11.852 & 0.002 & 10.961 & 0.002 & 0.044 & 0.005 & 1301 \\
\hline 8996.50775 & $2020-05-27.008$ & 13.508 & 0.007 & 11.896 & 0.004 & 10.937 & 0.005 & 9.932 & 0.011 & 0310 \\
\hline 8996.52131 & $2020-05-27.021$ & & & 11.874 & 0.006 & & & 9.919 & 0.010 & 0310 \\
\hline 8996.56371 & $2020-05-27.064$ & 13.395 & 0.010 & 11.866 & 0.004 & 10.954 & 0.004 & 9.927 & 0.006 & 1301 \\
\hline 8997.51757 & $2020-05-28.018$ & 13.481 & 0.007 & 11.870 & 0.006 & 10.927 & 0.006 & 9.914 & 0.013 & 0310 \\
\hline 8997.53240 & $2020-05-28.032$ & 13.411 & 0.010 & 11.839 & 0.005 & 10.914 & 0.003 & 9.883 & 0.005 & 1301 \\
\hline 8998.52015 & $2020-05-29.020$ & 13.480 & 0.010 & 11.828 & 0.005 & 10.979 & 0.011 & 9.923 & 0.012 & 0310 \\
\hline 9012.45686 & $2020-06-11.957$ & 13.416 & 0.007 & 11.804 & 0.004 & 10.835 & 0.005 & 9.883 & 0.015 & 0310 \\
\hline 9012.53045 & $2020-06-12.030$ & 13.339 & 0.010 & 11.758 & 0.004 & 10.827 & 0.003 & 9.824 & 0.008 & 1301 \\
\hline 9013.45632 & $2020-06-12.956$ & 13.421 & 0.006 & 11.791 & 0.005 & 10.857 & 0.007 & 9.888 & 0.013 & 0310 \\
\hline 9013.46770 & $2020-06-12.968$ & 13.377 & 0.006 & 11.766 & 0.003 & 10.861 & 0.008 & 9.902 & 0.009 & 1301 \\
\hline 9021.39395 & $2020-06-20.894$ & 13.432 & 0.004 & 11.816 & 0.006 & 10.882 & 0.008 & 9.882 & 0.014 & 1301 \\
\hline 9021.43568 & $2020-06-20.936$ & 13.440 & 0.008 & 11.834 & 0.005 & 10.858 & 0.006 & 9.883 & 0.012 & 0310 \\
\hline 9023.40886 & 2020-06-22.909 & 13.436 & 0.005 & 11.835 & 0.009 & 10.908 & 0.009 & 9.900 & 0.014 & 1301 \\
\hline 9023.43529 & $2020-06-22.935$ & 13.456 & 0.008 & 11.805 & 0.008 & 10.919 & 0.006 & 9.921 & 0.012 & 0310 \\
\hline 9024.42908 & 2020-06-23.929 & 13.483 & 0.007 & 11.876 & 0.005 & 10.891 & 0.005 & 9.910 & 0.013 & 0310 \\
\hline 9024.44579 & 2020-06-23.946 & 13.463 & 0.005 & 11.872 & 0.007 & 10.905 & 0.008 & 9.921 & 0.014 & 1301 \\
\hline 9027.46236 & $2020-06-26.962$ & 13.513 & 0.009 & 11.890 & 0.004 & 10.927 & 0.005 & 9.863 & 0.008 & 1301 \\
\hline 9030.42147 & $2020-06-29.921$ & 13.544 & 0.007 & 11.876 & 0.006 & 10.909 & 0.007 & 9.829 & 0.009 & 1301 \\
\hline 9036.39575 & $2020-07-05.896$ & & & 11.885 & 0.006 & & & 9.938 & 0.012 & 0310 \\
\hline 9037.40316 & $2020-07-06.903$ & 13.463 & 0.006 & 11.843 & 0.004 & 10.928 & 0.010 & 9.967 & 0.014 & 1301 \\
\hline 9038.39037 & $2020-07-07.890$ & 13.491 & 0.010 & 11.872 & 0.004 & 10.888 & 0.014 & 9.919 & 0.011 & 0310 \\
\hline 9039.38832 & $2020-07-08.888$ & 13.472 & 0.009 & 11.842 & 0.005 & 10.922 & 0.006 & 9.929 & 0.012 & 0310 \\
\hline 9043.37997 & $2020-07-12.880$ & 13.427 & 0.010 & 11.811 & 0.004 & & & 9.883 & 0.013 & 0310 \\
\hline 9056.37435 & $2020-07-25.874$ & & & 11.758 & 0.008 & & & 9.827 & 0.014 & 0310 \\
\hline 9056.37609 & $2020-07-25.876$ & 13.358 & 0.004 & 11.789 & 0.003 & 10.875 & 0.007 & 9.913 & 0.011 & 1301 \\
\hline 9058.38020 & $2020-07-27.880$ & 13.407 & 0.015 & 11.814 & 0.007 & 10.826 & 0.008 & 9.881 & 0.010 & 0310 \\
\hline 9067.36391 & $2020-08-05.864$ & 13.513 & 0.013 & 11.850 & 0.009 & & & 9.941 & 0.011 & 0310 \\
\hline 9068.35588 & $2020-08-06.856$ & 13.484 & 0.012 & 11.866 & 0.004 & 10.926 & 0.005 & 9.879 & 0.007 & 1301 \\
\hline
\end{tabular}

system via the transformations calibrated by Munari et al. (2014). Our photometry of 2SXPS is listed in Table 1. The quoted errors are the quadratic sum of the Poissonian error on the variable and the error in the transformation to the standard system via the above color equations.

\subsection{Spectroscopy}

Low resolution spectra of 2SXPS have been obtained with a Shelyak LHIRES spectrograph $+300 \mathrm{ln} / \mathrm{mm}$ grating mounted on the 0.50 Ritchey-Chretien tele- 
scope operated for ANS Collaboration in Stroncone (TR, Italy). The CCD Camera is an ATIK $460 \mathrm{EX}(2749 \times 2199$ pixels, $4.5 \mu \mathrm{m}$ in size $)$. A slit width of $30 \mu \mathrm{m}$ $(=2.0 \mathrm{arcsec})$ has been adopted. Table 2 presents a log of these low-res observations.

Echelle high resolution spectra of 2SXPS have been obtained with the $0.84 \mathrm{~m}$ telescope operated by ANS Collaboration in Varese (Italy), and equipped with a mark.III Multi-Mode Spectrograph from Astrolight Instruments. The detector is a SBIG ST10XME CCD camera $(2192 \times 1472$ array, $6.8 \mu \mathrm{m}$ pixel, KAF-3200ME chip with micro-lenses to boost the quantum efficiency). In the high resolution mode, an R2 Echelle grating of $79 \mathrm{ln} / \mathrm{mm}$ is used in conjunction with an equilateral $60^{\circ}$ prims made in high dispersion N-SF11 flint glass, for a final 18,000 resolving power with a 2 arcsec slit width. For 2SXPS observations a binning $2 \times 2$ was adopted leading to a resolving power 10,000.

The spectroscopic observations at both telescopes were obtained in long-slit mode, with the slit always rotated to the parallactic angle and widened to 2arcsec on the sky. All data have been similarly reduced within IRAF, carefully involving all steps connected with correction for bias, dark and flat, sky subtraction, wavelength calibration and heliocentric correction. The low-res spectra have been flux calibrated using similar observations of the nearby spectrophotometric standard HR 6698 observed immediately before or after 2SXPS.

Table 2. Log of spectroscopic observations of 2SXPS J173508.4-292958.

\begin{tabular}{|c|c|c|c|c|c|c|c|c|c|}
\hline \multicolumn{5}{|c|}{ low-resolution } & \multicolumn{5}{|c|}{ Echelle } \\
\hline \multicolumn{2}{|c|}{2020} & \multirow{2}{*}{$\begin{array}{c}\text { UT } \\
01: 38\end{array}$} & \multirow{2}{*}{$\begin{array}{c}\text { JD } \\
(-2450000)\end{array}$} & \multirow{2}{*}{$\begin{array}{l}\text { expt } \\
\text { (sec) } \\
9000\end{array}$} & \multicolumn{2}{|c|}{2020} & \multirow{2}{*}{$\begin{array}{c}\mathrm{UT} \\
02: 25\end{array}$} & \multirow{2}{*}{$\begin{array}{c}\text { JD } \\
(-2450000)\end{array}$} & \multirow{2}{*}{$\begin{array}{l}\text { expt } \\
\text { (sec) }\end{array}$} \\
\hline Apr & 24 & & & & Apr & 23 & & & \\
\hline \multirow{4}{*}{ May } & 03 & $00: 21$ & 8972.519 & 3600 & & 24 & $02: 30$ & 8963.604 & 3600 \\
\hline & 22 & 00:41 & 8991.534 & 5400 & May & 21 & $01: 28$ & 8990.561 & 4500 \\
\hline & 24 & $00: 15$ & 8993.516 & 4500 & Jul & 29 & $20: 58$ & 9060.374 & 3600 \\
\hline & 27 & $00: 14$ & 8996.515 & 2700 & Aug & 05 & $20: 40$ & 9067.361 & 4500 \\
\hline \multirow[t]{7}{*}{ Jun } & 11 & $23: 40$ & 9012.492 & 7200 & & 09 & $20: 15$ & 9071.344 & 3600 \\
\hline & 12 & $22: 50$ & 9013.457 & 7200 & & 20 & $19: 55$ & 9082.330 & 2700 \\
\hline & 20 & $20: 45$ & 9021.370 & 4500 & & & & & \\
\hline & 22 & $21: 02$ & 9023.382 & 3600 & & & & & \\
\hline & 23 & $22: 00$ & 9024.422 & 4500 & & & & & \\
\hline & 25 & $23: 15$ & 9026.474 & 7200 & & & & & \\
\hline & 29 & $21: 28$ & 9030.400 & 3600 & & & & & \\
\hline \multirow[t]{2}{*}{ Jul } & 12 & $21: 16$ & 9043.391 & 3600 & & & & & \\
\hline & 25 & $20: 21$ & 9056.352 & 4500 & & & & & \\
\hline \multirow[t]{2}{*}{ Aug } & 06 & $19: 48$ & 9068.329 & 3600 & & & & & \\
\hline & 20 & 19:07 & 9082.299 & 2700 & & & & & \\
\hline
\end{tabular}




\section{Results}

\subsection{Spectral classification, energy distribution, and radial velocities}

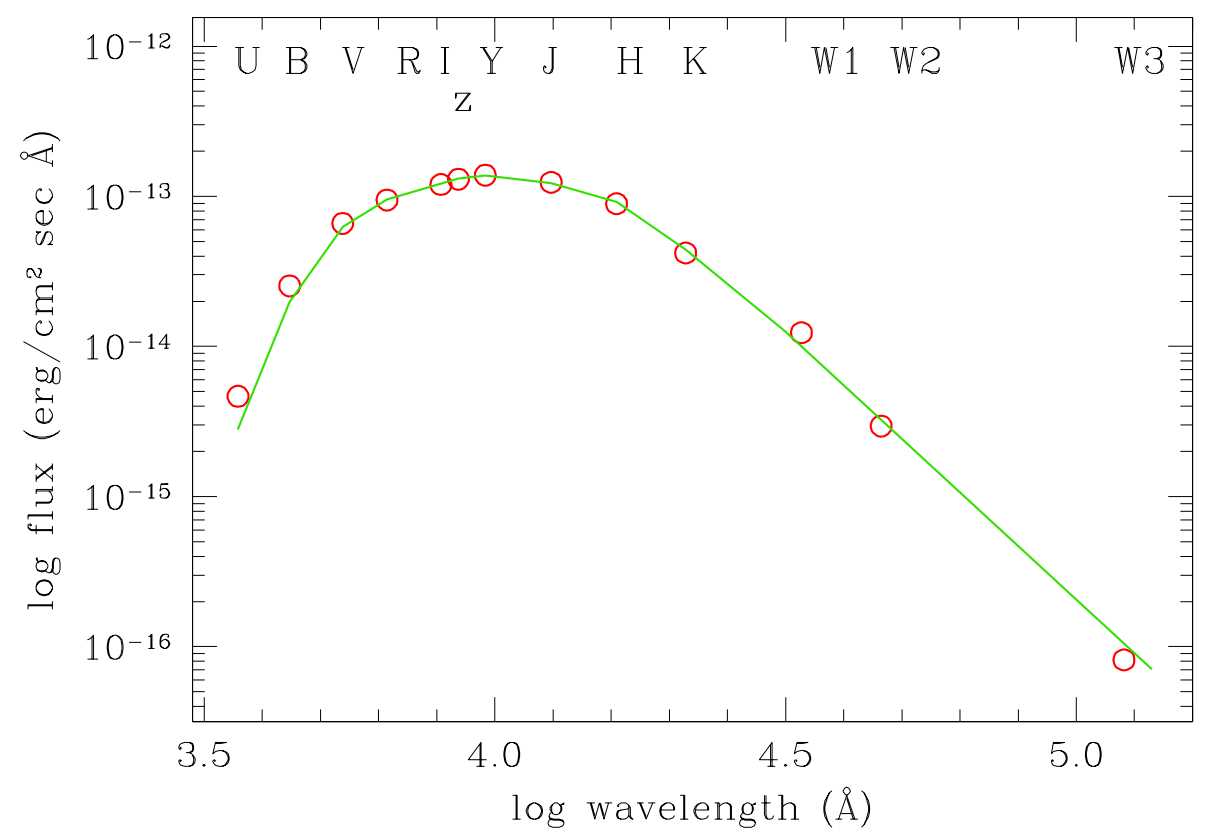

Figure 1. The observed energy distribution of 2SXPS J173508.4-292958 from our BVRIzY observations complemented with JHKs from 2MASS (Cutri et al. 2003), W1,W2,W3 from AllWISE (Cutri et al. 2014), and U band from Heinke et al. (2020). The green line is the intrinsic energy distribution of a K4III star from Koornneef (1983), Cousins (1980) and Fitzgerald (1970) subjected to an $E_{B-V}=0.375$ following the reddening law of Fitzpatrick (1999).

Even if projected close to the Galactic Center, 2SXPS is in the foreground at $1.50 \mathrm{kpc}$ distance according to Gaia eDR3 parallax 0.6685 \pm 0.0197 mas (Gaia Collaboration 2020). The distance would be minimally affected, to $1.53 \mathrm{kpc}$, by the application of the mean -0.017 mas offset derived by Lindegren et al. (2020) to affect globally the eDR3 parallaxes (they reported this bias to depend in a non-trivial way on - at least - the magnitude, colour, and ecliptic latitude of the source, with different dependencies that apply to the five- and six-parameter solutions in eDR3).

From Green et al. (2019) 3D extinction maps, 2SXPS should suffer from a modest $\mathrm{E}(\mathrm{B}-\mathrm{V})=0.375$. We have dereddened accordingly our BVRI photometry combined with 2MASS JHKs data and found an excellent match with the spec- 
tral energy distribution of a K4III giant (Fig. 1). Fitting instead with K3III or K5III distributions would require to change $\mathrm{E}(\mathrm{B}-\mathrm{V})$ to 0.49 or 0.28 , respectively. Combining distance, reddening and observed $\mathrm{V}=11.825$, an absolute magnitude $\mathrm{M}(\mathrm{V})=-0.55$ is derived, intermediate between K4III and K4III/II luminosity classes for which Sowell et al. (2007) list $\mathrm{M}(\mathrm{V})=+0.20$ and $\mathrm{M}(\mathrm{V})=-1.00$, respectively.

The AllWISE mid-IR data in Fig. 1 exclude the presence of circumstellar dust warmer than $\sim 200 \mathrm{~K}$. Any remnant blown-off by the progenitor of the present-day degenerate companion to the K4III has therefore already dispersed at a great distance from the central binary.

The SED in Fig. 1 is suggestive of an excess flux at the shortest wavelengths, amounting to $0.29 \mathrm{mag}$ at $\mathrm{U}$ band. A word of caution is however in order considering that the U-band Swift observation from Heinke et al. (2020) in Fig. 1 is not contemporaneous with the BVRIzY data, and 2SXPS is moderately variable (see below). So the apparent excess at the shortest wavelengths of Fig. 1 may be (in part or entirely) an artifact. Such an ultraviolet excess is frequently seen in symbiotic binaries of the accretion-only variety, and probably originates from the accretion disk around the companion to the cool giant (Mukai et al. 2016, Munari et al. 2020b). A significant contribution to the UV excess can be also provided by nebular emission from ionized gas located somewhere else in the binary system (Skopal 2005a,b), ionized by either the hard radiation emitted from the accretion disk and its central star or from some other mechanism, including wind collision. For simplicity, in this paper we attribute the UV excess to the accretion disk, including cumulatively in it also the contribution by any nebular region distinct from the disk itself.

The K4III classification for 2SXPS agrees with our low resolution spectra. Their average is plotted in Fig. 2 and compared with spectra of standards for spectral types K3III, K4III and K5III selected from the compilation by Yamashita and Nariai (1977) and observed along 2SXPS with the same spectrograph set-up. These nearby K giants trace the metallicity and chemical partition of the Solar neighborhod. The presence of $\mathrm{H} \alpha$ in emission in 2SXPS is rather obvious. The very large airmass affecting all our observations of 2SXPS marks the spectrum in Fig. 2 with signatures of telluric absorption red-ward of the $\mathrm{NaI}$ doublet at $5893 \AA$, the strongest feature being the $\mathrm{O}_{2}$ band at $6285 \AA$. The BaII feature at $6496 \AA$ shows the same strength in 2SXPS and the field K giants of Fig. 2, suggesting a normal abundance (for a discussion of Barium enhanced stars vs. symbiotic stars see Jorissen 2003).

Radial velocities have been obtained from the Echelle spectra by crosscorrelation (fxcor task in IRAF) with spectra of the IAU radial velocity standard HR 6859 obtained immediately before and after those of 2SXPS on each visit. This standard is rather convenient: it is located a short angular distance from 2SXPS at $\mathrm{RA}=18: 21: 48 \mathrm{DEC}=-29: 49: 18$ (minimizing differential spectrograph flexures), it is very bright at $\mathrm{V}=2.7 \mathrm{mag}$, and its K2III spectral type is an excellent proxy for that of 2 SXPS. We adopted the $-20.0 \pm 0.0 \mathrm{~km} \mathrm{~s}^{-1}$ heliocentric 


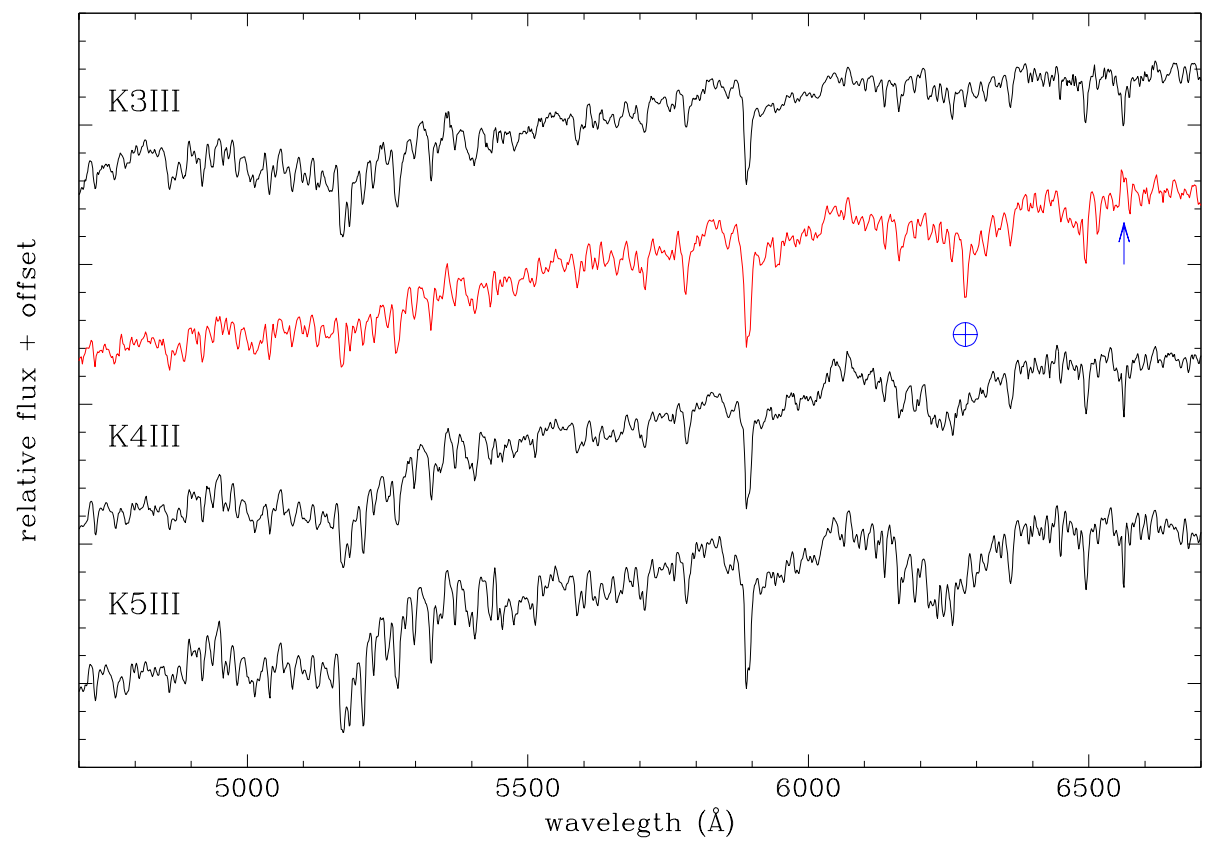

Figure 2. The low resolution spectrum of 2SXPS J173508.4-292958 compared with those of K3III, K4III and K5III spectral standards observed along with it. The strong telluric $\mathrm{O}_{2}$ band at $6285 \AA$ is marked, and the arrow points to $\mathrm{H} \alpha$ in obvious emission.

Table 3. Heliocentric radial velocities of the K-giant in 2SXPS J173508.4-292958 obtained with the Varese $0.84 \mathrm{~m}$ telescope.

\begin{tabular}{llcl}
\hline \hline & date & $\begin{array}{c}\mathrm{RV} \odot \\
(\mathrm{km} / \mathrm{s})\end{array}$ & $\begin{array}{l}\text { err } \\
(\mathrm{km} / \mathrm{s})\end{array}$ \\
\hline 2020 & Apr 23/24 & -11 & 1.5 \\
2020 & May 21 & -15 & 3 \\
2020 & Aug 05 & -13 & 1.5 \\
2020 & Aug 09 & -12 & 1.5 \\
2020 & Aug 20 & -12 & 2 \\
\hline \hline
\end{tabular}

velocity listed in the "Standard Radial Velocity Stars" section of the Nautical Almanac. The epoch radial velocities so obtained for 2SXPS are listed in Table 3 . They are fully compatible with a constant heliocentric radial velocity of $-12 \pm 1 \mathrm{~km} \mathrm{~s}^{-1}$ for 2 SXPS during the four months span of our observations. This suggests that the orbital period is either rather long (and as a consequence the amplitude of orbital motion quite small) and/or the viewing angle to 2SXPS 
is oriented high above the orbital plane. The tangential velocity of 2SXPS is similarly low at $-26 \mathrm{~km} \mathrm{~s}^{-1}$ (from Gaia eDR3 parallax and proper motions), for a combined space velocity of $29 \mathrm{~km} \mathrm{~s}^{-1}$.

\subsection{The variable and structured emission in $\mathrm{H} \alpha$}

Supporting the association of the K4III giant with the X-ray source 2SXPS is the presence on optical spectra of emission in $\mathrm{H} \alpha$, not expected in normal and single K4III stars belonging to the field.

In the left panel of Fig. 3 we present small portions around $\mathrm{H} \alpha$ from a sample of our low-resolution spectra (those with the highest $\mathrm{S} / \mathrm{N}$ ). The flux and equivalent width of $\mathrm{H} \alpha$ are observed to change by a large amount over the course of our observations; no correlation is noted (see phases marked on the spectra) with the low-amplitude (0.12 mag), 38-day sinusoidal variability exhibited by 2SXPS, and presumably associated with the K4III giant (see sect. 3.4 an Eq. 1 below). The absence of correlation, and the low temperature characterizing a K4III giant, suggest the accretion disk around the companion as the origin of the $\mathrm{H} \alpha$ emission.

The $\mathrm{H} \alpha$ profile as recorded on our Echelle spectra is presented on the right panel of Fig. 3. It is composed by a broad emission, about $470 \mathrm{~km} \mathrm{~s}^{-1}$ of width at half intensity, on which it is superimposed a narrow absorption component (only slightly wider than the instrumental PSF), which heliocentric velocity averaged over all spectra is $-4 \mathrm{~km} \mathrm{~s}^{-1}$. While the observed velocity of the K4III giant is consistent with a constant value, the velocity of the narrow absorption component could vary over the range -10 to $0 \mathrm{~km} \mathrm{~s}^{-1}$. Our spectra lack however the $\mathrm{S} / \mathrm{N}$ required for a firm conclusion about that.

One thing about the narrow absorption seems however well established: its velocity is positive with respect to the K4III giant, so it seems unlikely it may form in the gentle wind out-flowing from it and engulfing the whole binary system. A typical shift $\Delta \mathrm{vel} \approx-10 /-20 \mathrm{~km} \mathrm{~s}^{-1}$ is generally observed in symbiotic stars for the narrow absorption superimposed to the $\mathrm{H} \alpha$ emission, matching the typical wind velocity of field cool giants (Munari et al. 2020b, Shagatova et al. 2020). This leaves open the possibility that the narrow absorption component seen in 2SXPS may form elsewhere in the binary system (as the accretion disk itself or an atmosphere engulfing it), or in alternative its appearance is spurious and the $\mathrm{H} \alpha$ profile is actually composed by two separate emission components. The latter could be expected for an accretion disk seen at high inclination angle (e.g. Horne and Marsh 1986), but this condition seems to contrast with the limited (or null) orbital motion exhibited by the K4III giant over the four months of our observations. 

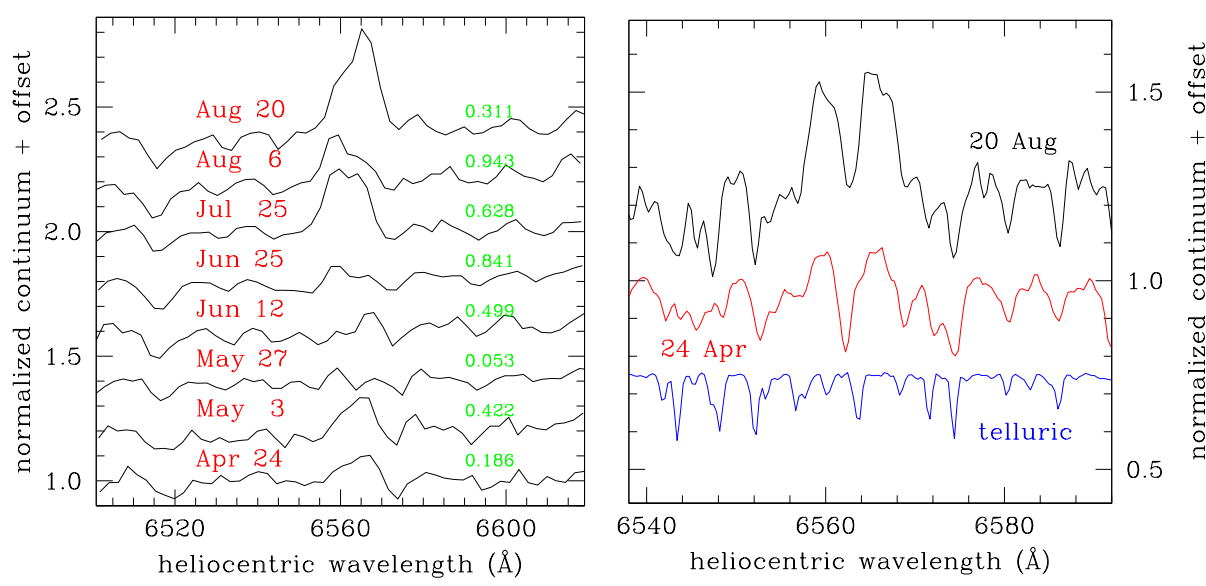

Figure 3. The variable $\mathrm{H} \alpha$ emission observed in 2SXPS J173508.4-292958 during 2020. Left panel: a strong variation in intensity in recorded on our low-res spectra. Right panel: on Echelle spectra the profile turns out to be characterized by a broad emission component with superimposed a narrow absorption. For reference, the spectrum at the bottom show the telluric absorption lines in the region as derived from observations of a telluric divider observed along the program star.

\subsection{No flickering observed}

We carried out a search for flickering at optical wavelengths as an indication of on-going accretion. To search for flickering we used a $50 \mathrm{~cm}$ telescope, with a 40arcmin corrected field of view and quality photometric filters, operated robotically for ANS Collaboration in Atacama (San Pedro Martir, Chile). We observed in $B$-band with 1-min integration time, with interspersed observations in $V$-band serving to construct the $B-V$ color base for the transformation from the instantaneous local photometric system to the Landolt's standard one. The same local photometric sequence and data reduction procedures as used for the long term $B V R I$ monitoring, were adopted.

About 50 field stars close on the image to 2SXPS, of a similar magnitude and well isolated from neighboring stars were also measured on all recorded images in exactly the same way as 2SXPS. The photometry of these 50 field stars was then inspected looking for those with a $B-V$ color as close as possible to 2SXPS. Four such stars were found, which serve as samplers of the observational noise above which the flickering has to be detected.

A first run, 70min in duration, was carried out from Sept 2.971 to 3.018 UT, 2020. The results are presented in Fig. 4: the dispersion of $B$-band measurements for 2SXPS is the same as for the four field stars of the same color and magnitude, and if any flickering is present, its amplitude does not exceed 0.005 mag. Other two runs, each one lasting 35min were carried out from Oct 24.980 to 25.003, 


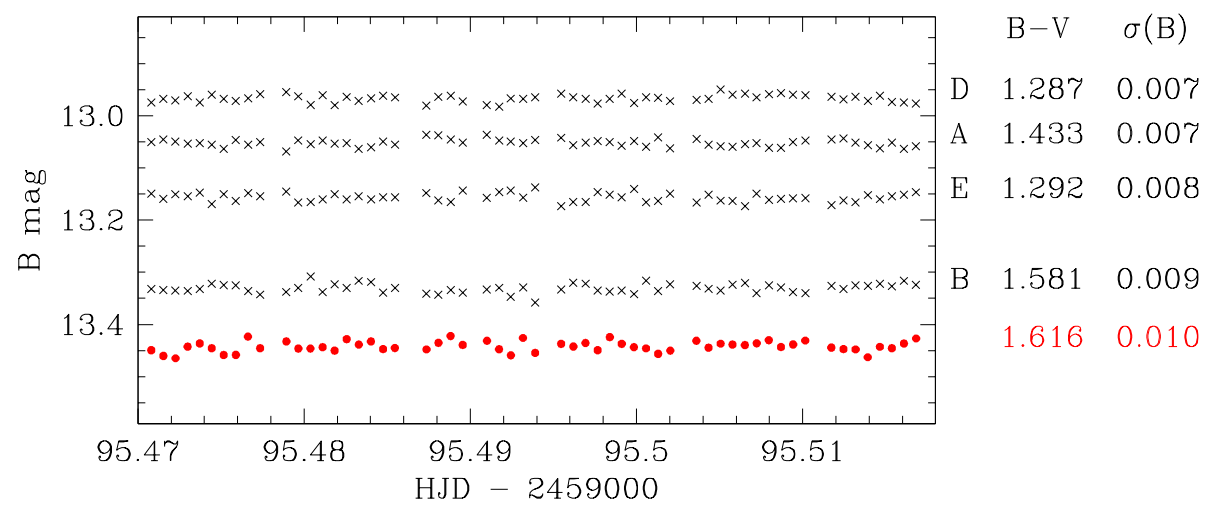

Figure 4. Results from a 70min run in $B$ band in search for flickering from 2SXPS J173508.4-292958 (red dots). The regular gaps in the data mark epochs when $V$-band exposures have been obtained to allow the transformation to the standard system. The corresponding data for four field stars of similar color and magnitude are presented as crosses. The progression from $\sigma=0.007$ for field star D to $\sigma=0.010$ for 2SXPS is accounted for by the $\Delta B \sim 0.5 \mathrm{mag}$ difference, and exclude the presence of any flickering with an amplitude in excess of 0.005 mag.

and from Oct 26.981 to 27.003 UT, 2020. They confirmed the findings for the early September run, with no flickering being detected.

We have seen above how large is the variability displayed by $\mathrm{H} \alpha$ emission. It probably parallels a similarly large variability of the accretion rate onto the compact object in 2SXPS. Therefore, the absence of flickering on early September and late October observations does not necessarily imply it is always absent. Unfortunately, at the time of flickering observations, the object was no more observable from Italy, and therefore we have no contemporaneous spectroscopy to compare the absence of flickering to the amount of emission in $\mathrm{H} \alpha$.

\subsection{Photometric evolution}

Our $B V R I$ photometry from Table 1 is plotted in Fig. 5. The $V$ band light-curve is characterized by a mean 11.825 mag value, and a sinusoidal modulation with a period of 38 days and an amplitude of $0.12 \mathrm{mag}$, with the following ephemeris providing times of mimina:

$$
\min (\mathrm{V})=2458994.5( \pm 1)+38( \pm 0.5) \times E
$$

The colors do not appear to change along the cycle. As illustrated by the SED in Fig. 1, the emission at optical wavelengths is dominated by the K4III giant, so the sinusoidal variability probably originates from it, either in the form of some pulsation or the rotation of a spotted surface. 


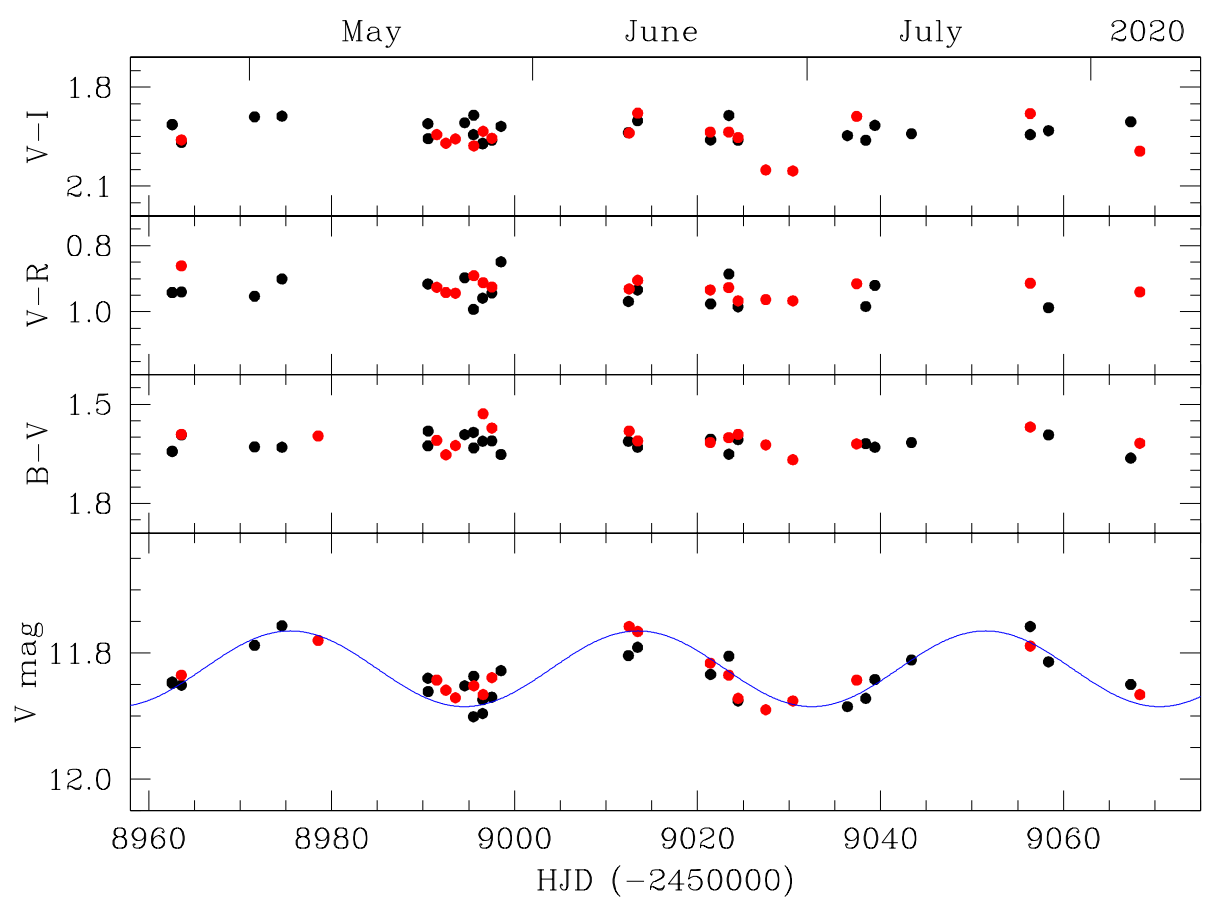

Figure 5. Our April-to-August 2020 light-curve of 2SXPS J173508.4-292958 built from data in Table 1 (red dots mark the observations obtained with ANS Collaboration telescope ID 0310, and black dots those with telescope ID 1301). The sinusoid going through the $V$-band data has minima according to Ephemeris (1), an amplitude of $0.12 \mathrm{mag}$ and a period of 38 days.

The sinusoidal variability could instead point to an ellipsoidal deformation of the K4III, should the latter fill its Roche lobe, in which case the orbital period would be twice the photometric one, or 76 days. The typical mass of a field K4III is listed as $1.2 \mathrm{M}_{\odot}$ by Drilling and Landolt (2000). Assuming $1.0 \mathrm{M}_{\odot}$ for the companion, this leads to a semi-major axis for the binary system of $98 \mathrm{R}_{\odot}$. The relation between mass ratio $q$, orbital separation $a$, and Roche lobe radius $R_{R L}$ as given by Eggleton (1983) is

$$
\frac{R_{R L}}{a}=\frac{0.49 q^{2 / 3}}{0.6 q^{2 / 3}+\ln \left(1+q^{1 / 3}\right)}
$$

and returns a radius of $39 \mathrm{R}_{\odot}$ for the K4III, twice the tabulated values of $\sim 20 \mathrm{R}_{\odot}$ (Drilling and Landolt 2000). The discrepancy would persist also for different assumption on the mass of the companion: $0.5 \mathrm{M}_{\odot}$ leads to $41 \mathrm{R}_{\odot}$ and 
$2.5 \mathrm{M}_{\odot}$ to $37 \mathrm{R}_{\odot}$. Therefore, the origin of the observed sinusoidal light-curve is probably other that Roche-lobe filling.
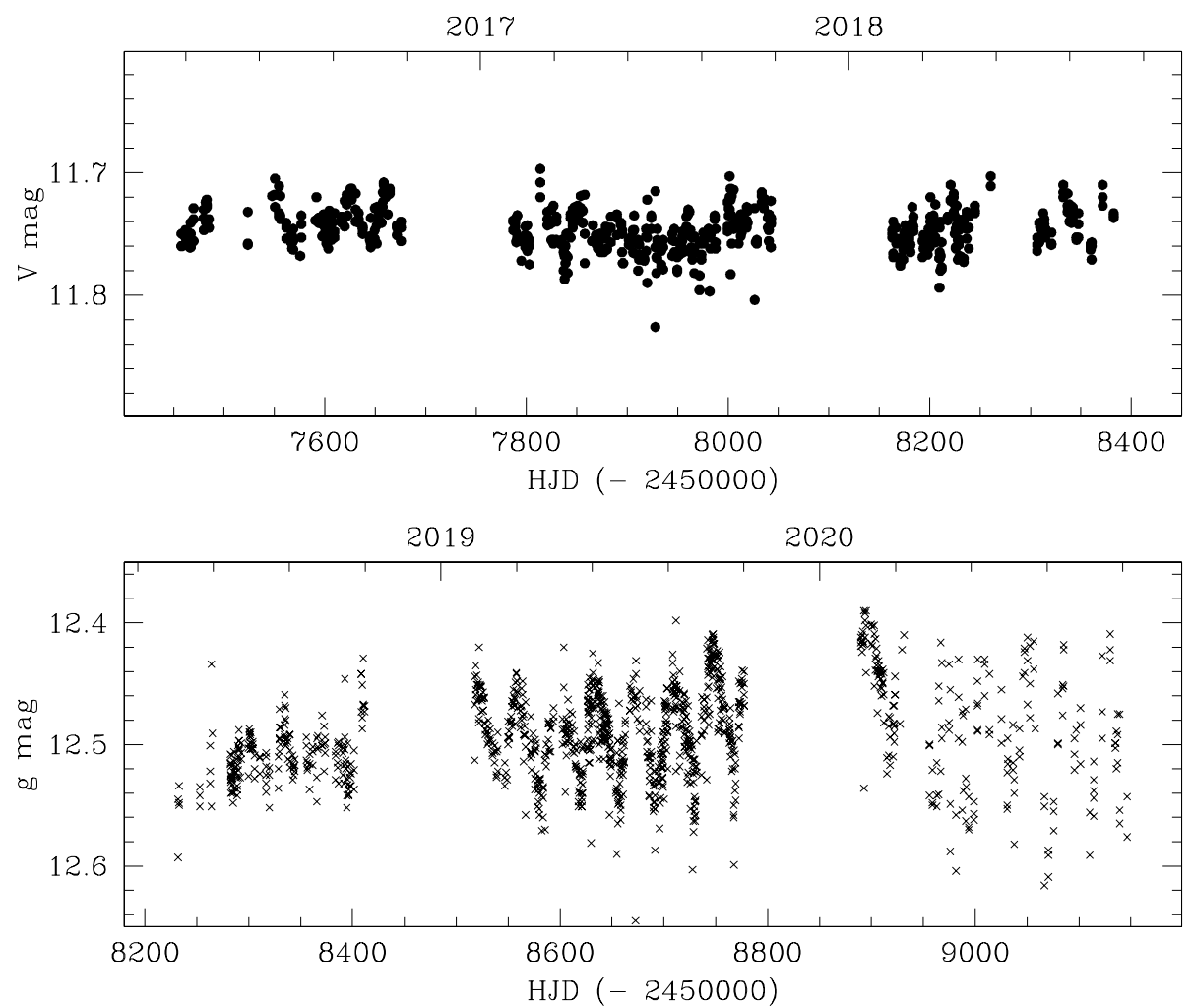

Figure 6. Long-term light-curve of 2SXPS J173508.4-292958 built from ASAS-SN sky-patrol data in $V$ and $g$ bands.

In support of this conclusion comes the absence of detectable radial velocity changes in Table 3. A 76-day period would correspond to an orbital velocity of the K4III of $30 \mathrm{~km} \mathrm{~s}^{-1}$ for a $0.5 \mathrm{M}_{\odot}$ companion and $38 \mathrm{~km} \mathrm{~s}^{-1}$ for $2.5 \mathrm{M}_{\odot}$. The maximum photometric amplitude (for edge-on systems) of the ellipsoidal modulation is $0.25 / 0.30 \mathrm{mag}$. An amplitude of $0.12 \mathrm{mag}$ for 2SXPS requires a significant orbital inclination, such that the amplitude of radial velocity variations would be at least $10 \mathrm{~km} \mathrm{~s}^{-1}$, incompatible with results from Table 3 .

The nail in the coffin about ellipsoidal modulation is however the fact that the sinusoidal variability is not always present but highly variable with time, as discussed in the following section. 


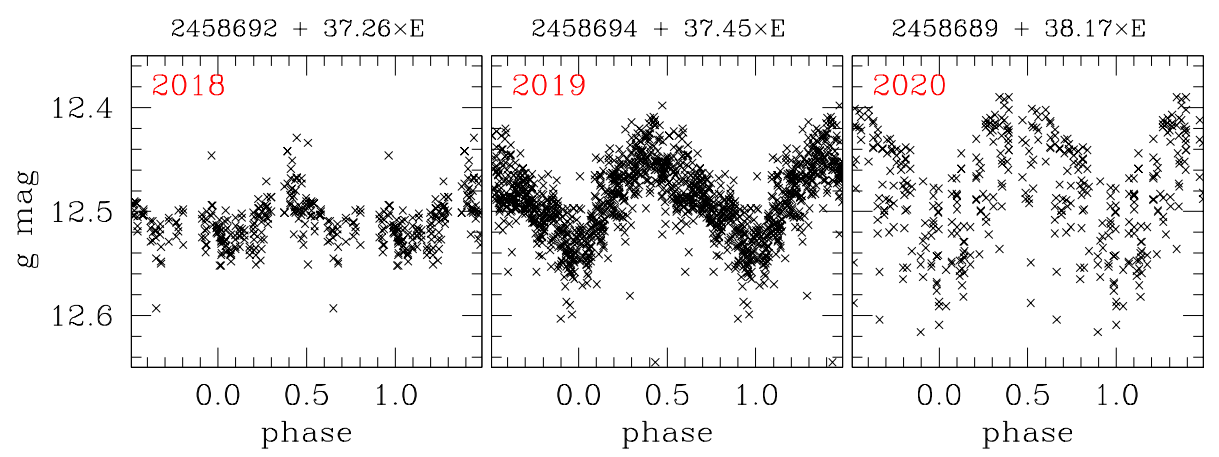

Figure 7. Phased light-curves for the modulated part of Figure 6.

\subsection{Long-term light-curve}

To reconstruct a longer term light-curve of 2SXPS we have downloaded ASASSN all-sky patrol data (Shappee et al. 2014, Kochanek et al. 2017) and plotted them in Fig. 6. 2SXPS was observed by ASAS-SN with a $V$-filter over 2016-2018 and with a $g$-filter during 2018-2020.

The median value of the 2016-2018 ASAS-SN $V$ band data in Fugure 6 is 0.076 mag brighter than our data in 2020. We do not regard this as significative: ASAS-SN data are just differential magnitudes with respect to not-transformed data for field stars, while our photometry is fully transformed to Landolt's system of equatorial standards (cf. sect. 2.1). The rather red color of 2SXPS, much redder than typical field stars, can easily account for the offset affecting ASASSN data.

The ASAS-SN light-curve in Fig. 6 shows a stable 2SXPS during 2016-2018, and appearance of the sinusoidal modulation only in 2019, which extended to 2020 with a lower degree of coherence. The phased light-curves of 2SXPS from ASAS-SN data for 2018, 2019 and 2020 is presented in Fig. 7, with the bestfit ephemeris given at the top of the panels. The differences between them are marginally within uncertainties.

While certainly arguing against an interpretation in terms of ellipsoidal distortion of the K4III giant, the light-curves of Fig. 5, 6 and 7 do not disentangle a specific cause for the variability observed in 2SXPS, although some kind of pulsation could be a viable explanation.

\section{Conclusions}

Our observations suggest 2SXPS to be a previously unknown symbiotic star, of the accreting-only variety (AO-SySt). The low space velocity, absence of cool circumstellar dust, solar-like metallicity and lack of enhancement in Barium set 
2SXPS aside from other symbiotic stars of the yellow class (i.e. those harbouring a cool giant of the F,G,K spectral types). As typical of AO-SySt systems, the signature for 2SXPS of on-going accretion onto a degenerate companion are feeble (weak and structure emission in $\mathrm{H} \alpha$, just a hint of near-UV excess emission) and highly variable in time, so that the AO-SySt nature may be not recognized at all times from optical observations alone. The star clearly deserves further observations, especially accurate radial velocities over a long time interval in order to derive the orbital period, orbital inclination and an estimate for the mass of the unseen companion.

\section{References}

Cousins A. W. J., 1980, SAAOC, 1

Cutri R. M., Skrutskie M. F., van Dyk S., Beichman C. A., Carpenter J. M., Chester T., Cambresy L., et al., 2003, yCat, II/246

Cutri R. M., et al., 2014, yCat, II/328

Drilling J. S., Landolt A. U., 2000, in Allen's Astrophysical Quantities, 4th ed., A.N. Cox ed., pag. 381, Springer-Verlag (New York)

Eggleton P. P., 1983, ApJ, 268, 368. doi:10.1086/160960

Fitzgerald M. P., 1970, A\&A, 4, 234

Fitzpatrick E. L., 1999, PASP, 111, 63. doi:10.1086/316293

Gaia Collaboration, 2020, yCat, I/350

Green G. M., Schlafly E., Zucker C., Speagle J. S., Finkbeiner D., 2019, ApJ, 887, 93. doi:10.3847/1538-4357/ab5362

Heinke C. O., Rivera Sandoval L., Maccarone T. J., Kennea J., Bahramian A., Strader J., Sivakoff G. R., 2020, ATel, 13648

Henden A. A., Templeton M., Terrell D., Smith T. C., Levine S., Welch D., 2016, yCat, II/336

Horne K., Marsh T. R., 1986, MNRAS, 218, 761. doi:10.1093/mnras/218.4.761

Jorissen A., 2003, ASPC, 303, 25

Kochanek C. S., Shappee B. J., Stanek K. Z., Holoien T. W.-S., Thompson T. A., Prieto J. L., Dong S., et al., 2017, PASP, 129, 104502. doi:10.1088/1538$3873 /$ aa $80 d 9$

Koornneef J., 1983, A\&A, 500, 247

Lindegren L., Bastian U., Biermann M., Bombrun A., de Torres A., Gerlach E., Geyer R., et al., 2020, arXiv, arXiv:2012.01742

Mukai K., Luna G. J. M., Cusumano G., Segreto A., Munari U., Sokoloski J. L., Lucy A. B., et al., 2016, MNRAS, 461, L1. doi:10.1093/mnrasl/slw087 
Munari U., 2019, in The Impact of Binary Stars on Stellar Evolution, G. Beccari and M.J. Boffin eds., Cambridge Univ. Press., Cambridge Astrophysical Series vol. 54, pag. 77 (arXiv:1909.01389)

Munari U., Henden A., Frigo A., Dallaporta S., 2014, JAD, 20, 4

Munari U., Valisa P., Dallaporta S., Vagnozzi A., Andreoli V., Castellani F., 2020a, ATel, 13660

Munari, U., Traven, G., Masetti, N. et al., 2020, MNRAS, submitted

Shagatova N., Skopal A., Shugarov S. Y., Komžík R., Kundra E., Teyssier F., 2020, arXiv, arXiv:2012.08417

Shappee B. J., Prieto J. L., Grupe D., Kochanek C. S., Stanek K. Z., De Rosa G., Mathur S., et al., 2014, ApJ, 788, 48. doi:10.1088/0004-637X/788/1/48

Skopal A., 2005a, A\&A, 440, 995. doi:10.1051/0004-6361:20034262

Skopal A., 2005b, ASPC, 330, 463

Sowell J. R., Trippe M., Caballero-Nieves S. M., Houk N., 2007, AJ, 134, 1089. doi:10.1086/520060

Yamashita Y., Nariai K., 1977, An Atlas of Representative Stellar Spectra, University of Tokyo Press 